gewidmet, welcher toxikologische Effekte, arbeitsmedizinische Fragen und Umweltaspekte explizit ansprach.

Auf der Podiumsdiskussion zu Chancen und Risiken verschiedener NanotechnologieLinien wurden die Standpunkte von den Referenten sachlich vertreten. Der Vertreter von Greenpeace (D. Parr) forderte nicht - wie andere NGO an anderer Stelle - ein generelles Moratorium für die Herstellung nanotechnologischer Produkte, sondern empfahl verstärkte Untersuchungen in solchen Bereichen, welche dispergierende Nanomaterialien nutzen. Diese zukünftig notwendige Begleitforschung wurde ebenfalls von den anwesenden Vertretern aus Industrie und Politik angeregt. M.C. Roco (NSF) führte an, dass kurzfristig ein hypeartiges Verhalten der Investoren und auch der Presse zu erkennen sei, aber langfristig die Nanotechnologie doch eine immense technologische Chance darstelle. „Overhyping“ wurde speziell von A. Gutsch (Degussa) als gefährlich für die letztendliche Verwertung der Ergebnisse eingeschätzt. Daher sei eine offene Informationspolitik aller Beteiligten anzuraten. Absehbare Risiken müssten adressiert werden, wobei nicht nur die direkten der Toxizität von Partikeln, sondern auch die möglichen gesellschaftlichen Veränderungen, welche sich evolutionär einschleichen könnten, eine Rolle spielen sollten. Bei der Diskussion der möglichen Implikationen sollte vom Problem kommend diskutiert werden, nicht generell von den technologischen Möglichkeiten aus. Auch die Frage des Risikos, falls man etwaige Risiken als Nation nicht auf sich nehmen wollte, wurde gestellt.

Beim Vergleich der verschiedenen internationalen Förder- und Umsetzungsstrategien konnten nur im Bereich Öffentlichkeitsarbeit und bzgl. erforderlicher Reaktionen auf Interessensgemeinschaften zum Stopp nanotechnologischer Arbeiten erhebliche Unterschiede festgestellt werden. Im asiatischen Raum sind derzeit kaum ethisch und soziologisch begründete Hindernisse für die Nanotechnologie festzustellen. Ansonsten werden weltweit wohl ähnliche Fragestellungen (Erkenntnisgewinn, Umsetzungsgeschwindigkeit für die Produktgenerierung, Ausbildungsfragen, Langfristmärkte, Infrastrukturen, Kooperationsformen) bei der F\&E-Förderung im Nanotechnologiebereich adressiert, jedoch mit unterschiedlicher Gewichtung und
Strategie. Ob die Grundlagenerarbeitung in den USA, die Industriekooperation in Europa oder die firmeninterne Forschung in Japan die erfolgreichere Strategie darstellen, wird sich wohl erst in der Zukunft zeigen.

Insgesamt hat sich die strategische Diskussion von Wertschöpfungsketten bei der nanoDE als eine wichtige Informationsplattform neben den wissenschaftlichen Präsentationen erwiesen.

Für weitere Informationen siehe

http://www.nanoDE.de sowie

http://www.nano2004.org.

\section{Raum für Nachhaltigkeit. Zur Kontextualisierung des Leitbilds}

\author{
Leipzig, 17. - 18. Juni 2004
}

\section{Tagungsbericht von Dieter Rink, Umwelt- forschungszentrum Leipzig-Halle}

Nachhaltigkeit hat Konjunktur, möchte man angesichts der Allgegenwart des Leitbilds in Politik und Gesellschaft meinen. Fragt man die jeweiligen Akteure jedoch nach ihrem Verständnis von Nachhaltigkeit, dann ergibt sich ein reichlich dissonantes Bild. Und soll das Leitbild gar als Richtschnur für politisches Handeln dienen, dann wird klar, dass dieses „hehre Ziel“ erst einmal für den jeweiligen Anwendungszusammenhang ausbuchstabiert und übersetzt werden muss, ehe an eine Nachhaltigkeits-orientierte Politik auch nur zu denken ist. Eine bedeutende Rolle bei der Anpassung des Leitbilds an konkrete räumliche, zeitliche, soziale und politische Gegebenheiten kann die Entwicklung und Anwendung regionaler und kommunaler Berichtssysteme spielen: Die Fokussierung auf einen bestimmten Analyseraum und die Kooperation von Wissenschaft mit Politik und Verwaltung erzwingen förmlich die Konkretisierung und Kontextualisierung von Nachhaltigkeitszielen.

Das BMBF hat vor drei Jahren einen Förderschwerpunkt eingerichtet, der die Erarbeitung und Implementierung von Berichtssyste- 
men unter dem Leitbild der Nachhaltigkeit für die regionale und kommunale Ebene zum Ziel hat. Die Tagung „Raum für Nachhaltigkeit. Zur Kontextualisierung des Leitbilds“ vom 17. - 18. Juni 2004 am Umweltforschungszentrum Leipzig-Halle (UFZ) widmete sich den Erfahrungen, die bei der Kontextualisierung von Nachhaltigkeit im Förderschwerpunkt „Konzeption und Erprobung problemorientierter regionale Berichtssysteme für eine nachhaltige Entwicklung“ (RBS) des BMBF bislang gesammelt wurden. Mit der Implementierung in Politik und Verwaltung und der Frage nach möglichen Steuerungswirkungen thematisierte sie zudem die politische Relevanz von Berichtssystemen. Ein weiteres Ziel der Tagung war die Einbettung der Projekte des Förderschwerpunktes in den deutschen Nachhaltigkeitsdiskurs.

\section{Konzepte und Verständnisse von Nach- haltigkeit}

Die Tagung gliederte sich in drei thematische Blöcke, die jeweils durch Impulsreferate von eingeladenen Experten eröffnet wurden. Im ersten Block ging es um Konzepte und Verständnisse von Nachhaltigkeit. Hier wurden die dominanten Konzepte der deutschen Nachhaltigkeitsdebatte mit den Nachhaltigkeitsverständnissen der RBS-Projekte und denen kommunaler Akteure kontrastiert.

Juliane Jörissen (Forschungszentrum Karlsruhe, ITAS) widmete sich in ihrem Impulsreferat den bekannten deutschen „Konzepten von Nachhaltigkeit" und versuchte, Grundlinien der Debatte nachzuzeichnen. Die deutsche Debatte zeige im Zeitverlauf den Trend zu zunehmender Komplexität. Dabei ließe sich folgende Entwicklung erkennen: Ausgangspunkt seien Ein-Säulen-Modelle gewesen (wie sie den Studien des Wuppertal-Instituts oder des Umweltbundesamtes zugrunde lagen), die den Vorrang von ökologischen Belangen postulierten. Dann seien Drei-Säulen-Modelle entwickelt worden, die eine Gleichrangigkeit von ökologischen, sozialen und ökonomischen Belangen vertreten (wie z. B. der Bericht der Enquete-Kommission „Schutz des Menschen und der Umwelt“ sowie die Studie „Arbeit und Ökologie“). Schließlich seien komplexe Konzepte ausgearbeitet worden, die dimensionsübergreifende Ziele formulierten und diesen Mindestanforderungen zuordneten.
Beispiele dafür seien die Nationale Nachhaltigkeitsstrategie der Bundesregierung und das Integrative Nachhaltigkeitskonzept der HelmholtzGemeinschaft (HGF). Man müsse jedoch sagen, so Jörissen, dass sich das Drei-Säulen-Modell in der deutschen Öffentlichkeit durchgesetzt habe und es schwer sei, andersartige Konzepte zu platzieren. In der Kritik an Nachhaltigkeitskonzepten würden folgende Punkte dominieren: deren Hyperkomplexität, die Verwässerung klarer Positionen, die Unterteilung in Dimensionen bzw. Säulen und die Ausrichtung an einzelnen Wissenschaftsdisziplinen (z. B. Ökologie, Ökonomie). Juliane Jörissen betonte, dass Nachhaltigkeit kein mehrdimensionaler Wunschzettel sei, sondern ein normatives Leitbild. Die Verbindung zwischen den globalen Normen und den jeweiligen örtlichen Verhältnissen müsse hergestellt werden, denn erst durch die Einbindung eines Teilraumes bzw. einer Region in den globalen Kontext werde ein Konzept zu einem Nachhaltigkeitskonzept.

Katja Huber und Dieter Rink (beide Umweltforschungszentrum Leipzig-Halle) stellten sodann in ihren Beiträgen die Nachhaltigkeitsverständnisse der RBS-Projekte sowie die ihrer Praxispartner vor, wobei sie sich auf eine Umfrage unter den Projekten des Förderschwerpunkts im Jahr 2003 bezogen. Hier zeige sich zwar auch das Bild der deutschen Debatte (wie von Jörissen skizziert), aber auch andere Konzepte. Überwiegend beziehe man sich auf gebräuchliche Nachhaltigkeitskonzepte, am ehesten Säulenkonzepte oder Kombinationen verschiedener Ansätze, führte Katja Huber aus, integrative Konzepte seien dagegen sehr selten. Bei der Rezeption des Leitbilds der Nachhaltigkeit bei den kommunalen Praxispartnern würden Skepsis und Ablehnung sowie Unbestimmtheit und Unsicherheit überwiegen, so Dieter Rink. Hinzu kämen Fragen nach dem Nutzen bzw. der Funktion des Leitbilds für die kommunale Praxis und es würden Schwierigkeiten gesehen, dies in den kommunalen Kontext einzuordnen. Auch bei den Praxispartnern dominierten Drei- bzw. Vier-Säulen-Konzepte. Auf der kommunalpolitischen Ebene werde Nachhaltigkeit dezidiert wirtschafts- bzw. arbeitsmarktpolitisch interpretiert und häufig recht plakativ verwendet: Als nachhaltig werde hier alles angesehen, was dauerhaft Arbeitsplätze schaffe. Daneben gebe es auf der Ver- 
waltungsebene ressortspezifische Zugänge, die zum Teil in Widerspruch zum kommunalpolitischen Verständnis stünden. Das entscheidende Defizit verortete Rink in der fehlenden Einbindung des Leitbilds Nachhaltigkeit in kommunale Leitbilder, Pläne und Beschlüsse. Hier sah er auch eine Aufgabe für die weitere wissenschaftliche Bearbeitung des Themas: es müsste geklärt werden, wie die Kommune Nachhaltigkeit in ihr Handeln implementieren könne.

\section{Kontextualisierung von Nachhaltigkeit}

Im zweiten Block „Kontextualisierung von Nachhaltigkeit“ sollten die Möglichkeiten der Kontextualisierung von Nachhaltigkeit mit den Strategien konfrontiert werden, die in den Projekten des Förderschwerpunkts zur Anwendung kommen.

Thomas Döring (Universität Kassel) und Stefan Heiland (Institut für Ökologische Raumentwicklung Dresden) gingen in ihrem Impulsreferat „Strategien der Kontextualisierung von Nachhaltigkeit“" auf die Potenziale von Nachhaltigkeitsindikatorensystemen ein. Dabei wiesen sie eingangs auf die Vielzahl von Kontextualisierungsdimensionen hin, wie z. B. Probleme, Themen, Ziele, Akteursgruppen oder Instrumente. Den Hintergrund ihrer Präsentation bildete eine eigene, im Auftrag des Umweltbundesamtes durchgeführte Studie zur vergleichenden Analyse von Nachhaltigkeitsindikatorensystemen auf kommunaler und regionaler Ebene. Ein wesentliches Ergebnis des Vergleichs sei, dass es viele Gemeinsamkeiten zwischen den Systemen gebe: Die Systeme seien häufig am Nachhaltigkeitsverständnis der Lokalen Agenda 21 ausgerichtet, die praktische Anwendbarkeit werde betont und die gleichen bzw. ähnliche Zielgruppen angesprochen (Politik, Verwaltung und Öffentlichkeit). Ziel sei meist nicht die konsequente bzw. konsistente Umsetzung eines Konzepts, sondern Information und Bewusstseinsbildung. Zudem sei die Indikatorenauswahl weitgehend an der Datenverfügbarkeit orientiert. Unterschiede bestünden in der Gliederung der Systeme, in den behandelten Themenbereichen und der Zahl der Indikatoren, aber auch im Umfang der Partizipation von Bürgern und gesellschaftlichen Gruppen sowie der inhaltlichen Verknüpfung der Daten. Dessen ungeachtet gebe es eine große Heterogenität der lokalen
Nachhaltigkeitsindikatorensysteme, was die beiden Autoren als Zeichen für eine starke Kontextualisierung deuteten. Der lokale Bezug werde bei der Entwicklung und beim Einsatz der Systeme betont, was auch zur Vernachlässigung konzeptioneller zugunsten pragmatischer Überlegungen führe. Die Auswertung der Themenfelder zeige, dass die Themen „Ökologie“ und „Soziales“ weit vor dem Thema „Wirtschaft“ rangierten und es insbesondere eine Ökologielastigkeit der Indikatoren gebe, die die ersten zehn Plätze belegen. Kommunale Nachhaltigkeitsindikatorensysteme würden - so ihr Fazit kaum genutzt. Wenn dann erfüllten sie in erster Linie eine Informationsfunktion und auch diese vorrangig für die Lokale Agenda. Die Nutzung für die politische Steuerung sei dagegen äußerst selten und sei vor allem nicht institutionalisiert. Die Ursachen für die geringe Nutzung lägen generell im Desinteresse am Thema Nachhaltigkeit und Nachhaltigkeitsindikatorensystemen, an ressortbezogenen Denk- und Verhaltensweisen, aber auch an Kapazitäts- und Finanzproblemen. Hinzu komme der umfassende Anspruch des Nachhaltigkeitspostulats, der mit dem fehlenden Bezug zum kommunalen Kontext kontrastiere. Die Indikatorensysteme seien zu unspezifisch für bestimmte Akteure und Funktionen. Außerdem sei auch die Frage der Anschlussfähigkeit der lokalen/regionalen Ebene an die übergeordnete (nationale) Ebene nicht geklärt.

Im Anschluss stellte Wibke Glismann (Universität Hamburg) das Projekt „Gesundheit als integrierendes Leitziel in der Konzeption und Erprobung eines regionalen Berichtssystems nachhaltiger Entwicklung“ vor. Hier werden in Kooperation mit 10 Städten in Ostdeutschland Berichtssysteme für die Bereiche Gesundheit, Soziales und Umwelt entwickelt. In Orientierung am Nachhaltigkeitspostulat des Brundtland-Berichts sollen unter Einbeziehung lokaler Agenda-Aktivitäten die genannten Themenbereiche vor einem gesundheitswissenschaftlichen Hintergrund integriert werden. Das Projekt folge dabei, so Glismann, einer pragmatischen Vorgehensweise im Hinblick auf die Auswahl von Indikatoren. Unter Rückgriff auf bereits vorhandene Indikatorensätze würde in einem Diskussionsprozess mit den beteiligten Städten ein Kernindikatorensatz von 35 Indikatoren nebst entsprechenden Zielbestimmungen benannt. Wibke Glismann zeigte sich optimistisch, dass es mit 
dem Projekt gelingen werde, das Thema „Gesundheit“ erfolgreich mit der Nachhaltigkeitsberichterstattung zu verknüpfen.

Antonina Bieszcz-Kaiser und Erhard Schreiber (Institut für Wirtschafts- und Sozialforschung Chemnitz e.V.) bezogen sich in ihrem Beitrag auf das Projekt „Konzeption und Erprobung eines Berichtssystems zur Beobachtung und Beschreibung von neuartigen Entwicklungen bei Beschäftigungsmaßnahmen auf der kommunalen Ebene unter dem Leitbild einer nachhaltigen Entwicklung“. Sie gingen der Frage nach, welchen Beitrag öffentlich geförderte Beschäftigung $\mathrm{zu}$ nachhaltiger kommunaler Entwicklung leistet. Ziel des Projekts sei die Erarbeitung eines Beobachtungs- und Berichtssystems als ein Instrument für die kommunalen Akteure, das ihnen hilft, ,eine sowohl der regionalen Arbeitsmarktlage noch adäquatere als auch nachhaltige kommunale Entwicklung befördernde Struktur der zum Einsatz kommenden arbeitsmarktpolitischen Instrumente zu bestimmen“. Es ginge darum, die Effektivität von Beschäftigungsmaßnahmen in diesem Sinne wesentlich zu erhöhen. Beschäftigungsmaßnahmen sollten nachhaltig dazu beitragen, das Arbeitsplatzdefizit zu verringern, die Beschäftigungsfähigkeit zu erhöhen und Zukunftsperspektiven zu eröffnen. Über die Reaktivierung brachliegenden Arbeitskräftepotenzials sollten wichtige kommunale bzw. regionale Aufgaben bearbeitet werden. Hierbei gelte es, ökologische, ökonomische und soziale Aspekte integrativ zu behandeln. Die Kommunen sollten mit dem Berichtssystem ein Instrument erhalten, das es ihnen gestatte, die Nachhaltigkeit von Beschäftigungsmaßnahmen zu bewerten.

In seinem Vortrag „Kommunale Problemfelder als Nachhaltigkeits-Kontext" umriss Gerhard Hartmuth (Umweltforschungszentrum Leipzig-Halle) die Kontextualisierungsstrategie des Projekts. Er charakterisierte die Ausgangslage bei der Entwicklung eines kommunalen Nachhaltigkeitsindikatorensystems als Akzeptanz- und Vermittlungsproblem. Die Haltung in der Verwaltung lasse sich pointiert in dem Satz zusammenfassen: „Wir haben hier eigentlich andere Probleme“. Daher seien die Operationalisierung und Kontextualisierung des Leitbilds im Sinne einer Anpassung an die lokalen Bedingungen zentrale Anforderungen. Im Projekt werde die Kontextualisierung über die Formu- lierung der vordringlichsten kommunalen Problemfelder geleistet. Diese würden mit den Nachhaltigkeitszielen eines integrativen Nachhaltigkeitskonzepts (des HGF-Konzepts; vgl. den Beitrag von Juliane Jörissen) verknüpft, bevor an der Schnittstelle von Problemfeldern und Nachhaltigkeitszielen Indikatoren identifiziert werden. Das integrative Nachhaltigkeitskonzept habe sich als Erfassungsraster und Bewertungsmaßstab für kommunale Probleme geeignet und so die Relevanz des Leitbilds für kommunale Problemfelder aufgezeigt. Dies habe zu einem umfassenderen Verständnis für Nachhaltigkeit bei den beteiligten kommunalen Akteuren geführt und die Akzeptanz des Leitbilds gestärkt. Eine Schwachstelle des Projekts sei die mangelnde Berücksichtigung aller kommunalen Handlungsfelder. Da die Methode zwangsläufig die Partizipation kommunaler Praxispartner beschränke, spiegelten sich die spezifischen Problemsichten einzelner Ämter bzw. Verwaltungsbereiche stärker wider als die anderer. Unterschiedliche Vorstellungen gäbe es bei der Gewichtung der Problemfelder. Die Praxispartner forderten die Priorisierung der Problemfelder entsprechend ihrer politischen Relevanz, die Wissenschaftler favorisierten dagegen deren Gleichbehandlung.

Am Beispiel der Region Mecklenburgische Seenplatte stellte Johann Käther (Fachhochschule Neubrandenburg) in seinem Vortrag „Nachhaltigkeitsindikatoren von unten“ die Frage, ob diese einen Beitrag zur regionalen Kontextualisierung von Nachhaltigkeit leisten können. Der Aufbau und die Etablierung von regionalen bzw. lokalen Nachhaltigkeitsberichtssystemen sei insbesondere mit dem Problem konfrontiert, was nachhaltige Entwicklung auf dieser Ebene bedeuten könne. Es mangele an der Anpassung des Leitbilds an die regionale Ebene und der Implementierung von Nachhaltigkeitsindikatorensystemen als Monitoring- und Steuerungsinstrument. Im Projekt „Freiwillige Selbstkontrolle Nachhaltigkeit“ werde Kontextualisierung durch die Problemorientierung (Einbeziehung lokaler bzw. regionaler Aspekte), die Akteursorientierung (Einbeziehung von Praxispartnern) sowie die Verknüpfung von Politik- bzw. Handlungsfeldern erreicht. Die Probleme der Kontextualisierung lägen etwa in den unterschiedlichen Nachhaltigkeitsverständnissen der Akteure, der Begrenztheit des Indika- 
torensatzes sowie den Defiziten bei der Verfügbarkeit. Außerdem gebe es unterschiedliche Bewertungsmaßstäbe und die Indikatoren seien das Ergebnis politischer Aushandlungsprozesse. Dadurch würden nur geringe Steuerungswirkungen entfaltet, die möglicherweise nicht überzeugend sind für eine dauerhafte Implementierung und Nutzung von Berichtssystemen.

Pamela Dorsch und Frauke Hoffmann (Technische Universität Berlin) präsentierten das Projekt „Nachhaltiger Tourismus in der Prignitz - Ein Informationssystem“. Im Projekt werde mit einem „projektbezogenen Grundverständnis" von Nachhaltigkeit gearbeitet, das sich auf die drei Dimensionen „Ökologie“, „Ökonomie“ und „Soziales“ (plus „Institutionelles“) beziehe. Die Kontextualisierung erfolge durch die Beteiligung der Akteure an allen Entwicklungsschritten: an der Stärken-SchwächenAnalyse des regionalen Tourismus und an der Analyse von Akteurskonstellationen. Daraus werde ein Zielsystem für nachhaltigen Tourismus entwickelt, das den Dimensionen zugeordnet sei und zu den Zielthemen jeweils Teilziele enthalte. Das Berichtssystem habe drei Funktionen: es biete Orientierungswissen, mache ein Kommunikationsangebot und beinhalte das eigentliche Monitoring mit einem regionalen Ziel- und Indikatorensystem. Kern des Projekts sei die Regionalisierung des Leitbilds mit dem Ziel der Steuerung der regionalen Entwicklung.

\section{Politische Implikationen von Nachhaltig- keits-Berichtssystemen}

Der dritte Block „Politische Implikationen von Nachhaltigkeits-Berichtssystemen“ wurde mit einem Impulsreferat zum derzeitigen Stand in Deutschland eingeleitet, an den sich Berichte aus der kommunalen Praxis anschlossen.

Ulrich Gehrlein (Institut für ländliche Strukturentwicklung Frankfurt/Main) gab in seinem Impulsreferat einen Überblick über die „Implementierung und Steuerungswirkungen von Nachhaltigkeitsberichtssystemen“. Es gebe etwa 250 Kommunen, in denen derartige Systeme angewendet würden - allerdings in ganz unterschiedlicher Form. Die Zielsetzung bestehe überwiegend in der Bestandsaufnahme bzw. Berichterstattung, der Bestimmung von Handlungsbedarf und der Öffentlichkeitsarbeit. Weit weniger jedoch würden damit Ziele wie die politische Entscheidungsunterstützung, die Steuerung des Verwaltungshandelns oder die Erfolgskontrolle der Zielumsetzung verfolgt. Infolgedessen hätten die Systeme zwar zur Bewusstseinsbildung in Politik und Verwaltung beigetragen, aber kaum zur Steuerung kommunaler Entwicklung. Die Ursachen dafür sah Gehrlein auch in der schwachen Kontextualisierung und mangelnden Implementierung von Berichtssystemen: Sie seien kaum in Arbeitsabläufe und Entscheidungsprozesse integriert, schlecht in das institutionelle Setting eingebettet und kaum mit Planungs- und Managementinstrumenten verknüpft. Aber auch auf der inhaltlich-konzeptionellen Ebene machte er Defizite aus: Die Indikatoren besäßen bislang nur eine eingeschränkte Handlungsrelevanz für die kommunale Praxis. Bisherige Nachhaltigkeitsindikatorensysteme würden überwiegend einem übergeordneten, querschnittsorientierten Kernindikatorenmodell entsprechen, aber wenig spezifisch für einzelne Steuerungsebenen sein. Hier stelle sich aber die Frage, ob die Gesamtentwicklung langfristig gesteuert werden solle oder konkrete Projekte? Wünschenswert seien aus dieser Perspektive funktionsspezifische Konzepte mit einer höheren Handlungsrelevanz. Dabei müssten insbesondere kommunale bzw. regionale Politikziele Aufnahme finden. Gehrlein meinte, dass sich die Berichtssysteme nur dann umfassend in die Kommunalpolitik bzw. -verwaltung implementieren ließen, wenn sie in das Neue Steuerungsmodell integriert würden. Nachhaltigkeitsindikatorensysteme hätten dann nämlich zentrale koordinierende Funktionen und würden als Controlling-Instrumente eines differenzierten Berichtssystems in Politik und Verwaltung eingesetzt. Daher seien die Systeme in diese Richtung zu entwickeln und Aspekten der Implementierung müsste in Zukunft viel mehr Aufmerksamkeit gewidmet werden.

Der nachfolgende Vortrag von Erwin Rothgang (Stadt Wuppertal) zeichnete den Prozess der Implementierung eines Nachhaltigkeitsberichtssystems in der Stadt Wuppertal nach. An diesem Beispiel werde sinnfällig, dass dieser Prozess (viel) Zeit brauche und von den Akteuren großes Durchhaltevermögen erfordere. In Wuppertal sei 1995 mit dem Agenda-Beschluss des Stadtrats der Startschuss gefallen und erst 2004 die strategische Steuerung unter dem Leitbild der Nachhaltigkeit mit Zielvereinbarungen, 
Kennzahlen und Indikatoren eingeführt worden. Rothgang wies in seiner engagierten Präsentation nachdrücklich darauf hin, dass der Implementierung viel Aufmerksamkeit gewidmet werde müsse und Politiker von Anfang an einzubinden seien.

Ein weiteres Beispiel für die „Dauerhafte Implementierung eines Nachhaltigkeitsberichtssystems" wurde von Annett Zimmermann (Stadt Güstrow) am Beispiel der Stadt Güstrow demonstriert. Ausgangspunkt für die Entwicklung eines Nachhaltigkeitsberichtssystems sei auch hier der Agenda-Prozess gewesen, der mit kommunalen Aktivitäten verknüpft worden sei. Es sei von Anfang an ein ganzheitlicher Projektansatz verfolgt worden, der die Vernetzung ökonomischer, ökologischer, sozialer und kultureller Aspekte vorsah. Innerhalb der Stadtverwaltung, so Zimmermann, sei der Umgang mit den Indikatoren inzwischen zum Alltagsgeschäft geworden und habe sich professionalisiert. Dies habe ein Bewusstsein für das Controlling der Stadt- und Wirtschaftsentwicklung geschaffen und diene der Kontrolle der eingesetzten Strategien und Maßnahmen. Allerdings seien die Indikatoren dadurch vorrangig Planungswerkzeuge geworden. Auf der politischen Ebene würde demgegenüber eher mit qualitativen Zielen und Strategien operiert als mit quantitativen Größen. Ungeachtet dieser Differenzen schätzte die Güstrower Planerin den Implementierungsprozess als erfolgreich ein.

\section{Nachhaltigkeitsberichtssysteme: Von der Kontextualisierung zur Implementierung?}

Die Tagung machte die breite Palette an Forschungsaktivitäten zum Thema sichtbar und gestattete eine Verortung der Projekte des RBSFörderschwerpunktes in diesem Feld. In Bezug auf das Thema der Tagung, die Kontextualisierung, wurde die Vielfalt der Strategien sichtbar, die von akteursbezogenen über thematische bis hin zu räumlichen und funktionsbezogenen Strategien reichen. In den Diskussionen wurde deutlich, dass für eine erfolgreiche Implementierung in der Verwaltung eine Kontextualisierung im Hinblick auf institutionelle Abläufe erforderlich ist. Dazu taugen die vorliegenden Indikatorensysteme nur bedingt, da sie in erster Linie für die (öffentliche) Information und Kommunikation entwickelt wurden und infolgedessen vor allem zur Bewusstseinsbildung beigetragen haben. Um sie aber in der kommunalen Praxis als Steuerungsinstrument nutzen zu können, bedarf es der Ausrichtung auf spezifische Berichtspflichten sowie kommunale Ziele und Beschlüsse. Selbst wenn das Leitbild der Nachhaltigkeit akzeptiert und ein kommunaler $\mathrm{Zu}$ gang dazu entwickelt ist, so muss ein entsprechendes Berichtssystem nicht unbedingt schon allein deshalb anschlussfähig an die kommunale Steuerungsphilosophie sein.

Neben dem breiten Überblick über die vorhandenen und die in den RBS-Projekten in Entwicklung befindlichen Berichtssysteme machte die Tagung auch Fortschritte sichtbar. Diese bestehen etwa in der mittlerweile erreichten Vielfalt der entwickelten Indikatorensysteme, in der Varianz der verwendeten Konzepte, in der Heterogenität der angewandten partizipativen Verfahren sowie in der stärkeren Ausrichtung auf spezifische Funktionen und Ziele. Es wurde aber auch deutlich, dass viele der entwickelten Systeme einmalige (Kraft)Akte darstellen, die in dieser Form nicht wiederholbar sind. Die beteiligten Akteure, gerade aus dem Umfeld der Lokalen Agenden, verfügen in der Regel nicht über die nötigen Ressourcen, um Berichtssysteme auf Dauer stellen zu können. Zugleich wurde deutlich, dass sich die bislang entwickelten Berichtssysteme innerhalb eines Rahmens bewegen: Es werden keine grundsätzlich neuen Systeme mehr entwickelt, sondern es werden überwiegend bekannte Konzepte herangezogen und vorliegende Indikatorensets werden nur noch wenig verändert. Die RBS-Projekte selbst bauen im Wesentlichen auf dem erreichten Stand der Indikatorenentwicklung auf und haben ihm nur partiell Neues hinzugefügt. Dafür sind sie stärker kontextualisiert, etwa durch ihre Ausrichtung auf die Bedürfnisse und Erwartungen der kommunalen Verwaltungspraxis oder die Einbettung in Formen der ämterübergreifenden Zusammenarbeit.

Abschließend wurde die Frage diskutiert, ob im Prozess der Implementierung in die kommunale Praxis schon der „Point of no return" erreicht sei. Eine Reihe von Teilnehmern bezweifelte dies mit Blick auf die bisher geringe Zahl an erfolgreichen Beispielen. Es blieb dabei offen, inwiefern die Implementierung an grundsätzlichen Hindernissen scheitert. Deutlich wurde, dass künftig die Beschäftigung - auch die 
wissenschaftliche Untersuchung - der Voraussetzungen, Bedingungen und Schritte bei der Implementierung der Berichtssysteme in das kommunale Verwaltungshandeln von zentraler Bedeutung für Fortschritte auf diesem Gebiet sein wird. Es sind auch weitere Anstrengungen nötig, um die Zugänglichkeit der Systeme für die Öffentlichkeit zu erhöhen. Die Anwendung bzw. Umsetzung der Berichtssysteme mittels elektronischer Software und ihre Einspeisung in kommunale Intra- bzw. Internetsysteme steht erst am Anfang. Die Diskussion endete eher mit offenen Fragen als mit Antworten - was beim derzeitigen Forschungsstand in diesem Feld auch nicht überraschend ist. Auf welche Ebene zielen kommunale Berichtssysteme, auf die der strategischen Kommunalpolitik, die des Verwaltungshandelns - auch einzelner Ressorts - oder die einzelner Projekte? Welche spezifischen Steuerungs- und Controlling-Funktionen können kontextualisierte Nachhaltigkeitsberichtssysteme haben? Usw. Engagiert wurde dafür gestritten, sich mit derartigen Berichtssystemen „nicht in die Historie schieben (zu) lassen“.

Die Beiträge der Tagung sollen in einem Band in der von Armin Grunwald und Bernd Hansjürgens herausgegebenen Reihe „Global zukunftsfähige Entwicklung - Perspektiven für Deutschland“ (edition sigma) publiziert werden.

\section{》)}

\section{Ökologische Ökonomie: eine neue Wissenschaft?}

Heidelberg, 6. - 8. Mai 2004

\section{Tagungsbericht von Fred Luks, Hamburger Universität für Wirtschaft und Politik, Pro- jekt NEDS - Nachhaltige Entwicklung zwi- schen Durchsatz und Symbolik}

Welche Funktion kann die Wissenschaft in einer sich wandelnden Welt haben, wenn das Ziel eine nachhaltige Entwicklung ist? Welche Rolle kann die Ökologische Ökonomie in diesem Zusammenhang spielen? Inwieweit ist sie eine „neue Wissenschaft“, die zur Lösung wichtiger Gegenwarts- und Zukunftsprobleme Beiträge leisten kann? Diese und andere durch das Leitbild „Nachhaltige Entwicklung“ provo- zierte Fragen an die Wissenschaft im Allgemeinen und die Ökologische Ökonomie im Besonderen waren Gegenstand der kleinen, thematisch breit angelegten und inhaltsreichen Tagung „Ökologische Ökonomie: eine neue Wissenschaft?“, die von der Vereinigung Ökologische Ökonomie (VÖÖ) vom 6.-8. Mai 2004 in Heidelberg ausgerichtet wurde.

Die Ökologische Ökonomie befasst sich mit Ganzheiten ebenso wie mit spezifischen wirtschaftlichen, sozialen und ökologischen Problemen. Sie sieht sich selbst als offenen Suchprozess, der zum Suchprozess „Nachhaltige Entwicklung“ praktikable Lösungen beitragen will. Diese normativ und im Hinblick auf die Vision einer nachhaltigen Entwicklung motivierten Charakteristika der Ökologischen Ökonomie als „neuer Wissenschaft“ erwiesen sich als guter Ausgangspunkt für einen drei Tage langen Diskussionsprozess, an dem sich über 30 WissenschaftlerInnen unterschiedlicher Disziplinen und unterschiedlicher Generationen intensiv beteiligten. Einmal mehr war der „Heidelberg-Spirit“ der Jahrestagung zu spüren - der intensive Gedanken- und Erfahrungsaustausch zwischen den Sitzungen war ebenso wichtig wie die „offziellen“ Plena und Arbeitsgruppen.

Eine zentrale Scharnierstelle für die Verbindung von Wissenschaft und Nachhaltigkeit ist die disziplinäre Organisation von Forschung und Lehre. Wenn die Wissenschaft nicht nur Teil der Probleme, sondern Teil der Lösungen sein will, muss sie eben diese Disziplinarität hinterfragen und sich auf Debatten zum Status von Mono-, Multi-, Inter- und Transdiziplinarität einlassen. Die Tatsache, dass ein analytisch, partikularisierend und disziplinär ausgerichteter Wissenschaftsbetrieb vor dem Hintergrund von Nachhaltigkeitsproblemen an Überzeugungskraft verliert, war ein wiederkehrendes Thema. Es gehört zu den Grundannahmen der Ökologischen Ökonomie, dass eine an Nachhaltigkeit orientierte Reform von Gesellschaft und Wirtschaft mit einer Reform der Art und Weise einhergehen muss, mit der Wissenschaft betrieben wird. $\mathrm{Zu}$ thematisieren ist in diesem Zusammenhang mithin „Kultur“ als Ganzes, und auch dies wurde auf der Tagung versucht. $\mathrm{Zu}$ fragen war also, wie eine neue Wissenschaft und eine neue Wissenschaftskultur aussehen können, und welche Bedeutung der Ökologischen Ökonomie in diesem Zusammenhang zukommt. Gegen- 